REVISTA CHILENA DE LITERATURA

Abril 2010, Número 76, 129 - 149

\title{
BERNE-COPENHAGUE-MADRID-PARÍS-SANTIAGO: INTERPOLACIONES RELATIVISTAS, VARIACIONES CUÁNTICAS E IMPACTOS CÓSMICOS EN ALTAZOR $(1919-1931)^{1}$
}

\author{
Scott Weintraub \\ The University of Georgia \\ seweint@uga.edu
}

RESUMEN / ABSTRACT

En este artículo para el número especial de Revista Chilena de Literatura que conmemora el bicentenario chileno, exploro múltiples conexiones literarias, científicas, y geográficas entre el poema Altazor de Vicente Huidobro y los contextos filosófico-científicos globales de las primeras décadas del siglo XX. Evalúo el impacto de una serie de caídas lingüísticas, críticas, alegóricas y gravitacionales en la trayectoria del "viaje en paracaídas" de Altazor, para volver a considerar, desde varias perspectivas críticas que tratan la "ilegible" y ambigua conclusión del poema, el impacto de un evento lingüístico en el campo de gravedad del poema. De esta manera, el artículo examina el imaginario científico que Altazor comparte con ciertos descubrimientos clave en la física teórica y experimental durante la primera parte del siglo XX, vinculando la escena poética vanguardista chilena y europea (Madrid-Paris-Santiago) con los avances científicos de la época (Berne-Copenhague). Dicha indagación histórico-contextual con el cambio de paradigma involucrado en el universo cuántico-relativista -contemporáneo a la fecha de composición del poema- exige una exploración de las maneras en que Altazor qua texto poético marca el paso discursivo entre las cosmovisiones newtonianas y cuánticas. El campo semántico de Altazor, examinado con respecto a estas cuestiones cosmológicas, demuestra cómo el poema largo de Huidobro marca un evento lingüístico y cósmico que, no obstante, es sin horizonte de expectativa y así radicalmente heterogéneo en su naturaleza -faceta del poema que subraya el tipo de variaciones y flujos cuánticos cuyos "senderos" no se pueden predecir con certidumbre.

1 Dedico este artículo a Luis Correa-Díaz, poeta y cosmonauta errante. 
Palabras clave: Vicente Huidobro, Altazor, poesía, física cuántica, relatividad.

In this article for the special issue of Revista Chilena de Literatura devoted to Chile's bicentennial, I explore multiple literary, scientific, and geographic connections between Vicente Huidobro's poem Altazor and the global philosophical-scientific contexts of the first few decades of the $20^{\text {th }}$ century. I consider the impact of a series of linguistic, critical, allegorical, and gravitational "falls" in the trajectory of Altazor's "viaje en paracaidas" in order to think through the impact of a linguistic event in the poem's gravitational field. By engaging myriad critical approaches to the poem's "illegible," ambiguous conclusion, I link the Chilean and European avant-garde poetic scenes (Madrid-Paris-Santiago) with the scientific imaginaries of important discoveries in theoretical and experimental physics in the early $20^{\text {th }}$ century (Berne and Copenhagen). I explore the ways in which Altazor in and of itself the marks the discursive passage between Newtonian and quantum cosmovisions vis à vis the historical context of the quantum/relativistic paradigm shift in physics that was contemporary to the poem 's composition. Altazor's meaning-making activities, read with respect to quantum and cosmological concerns, show how Huidobro's long poem traces the falling motion of a linguistic and cosmic event that, nevertheless, is horizon-less and radically heterogeneous in nature-a facet of the poem that is indicative of the kinds of quantum fluctuations whose "path" cannot be accurately predicted or described with total certainty or mastery.

KEY WORDS: Vicente Huidobro, Altazor, poetry, quantum physics, relativity.

El poema largo Altazor de Vicente Huidobro es una exploración vanguardista del lenguaje, la cual busca narrar una serie de caídas lingüísticas, críticas, alegóricas y gravitacionales para mapear la trayectoria del "viaje en paracaídas" del protagonista. Se podría situar o localizar el impacto de un evento significativo, desde el punto de vista referencial y discursivo, en las configuraciones teóricas de la caída y la gravedad, en medio de la poética celestial huidobriana íntimamente vinculada al espacio cósmico y los flujos lingüísticos que lo delimitan. A la luz de las deconstrucciones extra-territoriales altazorianas, varios estudiosos han descrito la reconfiguración poética de una caída "desde" la referencialidad del signo lingüístico como función de la caída material del lenguaje mismo. Una indagación crítica de dicha argumentación, partiendo del campo de las investigaciones cosmológicas, podría dirigirse a las estructuras referenciales fantasmáticas del poema para luego examinar cómo las múltiples, desarticuladas cadenas de significantes registran un evento celestial y cuántico sin posibilidad de ser previsto ni esperado, manifestado en el espacio textual de Altazor y particularmente en la radicalidad de los famosos gritos finales del poema. 
Este ensayo retoma el hilo de los posibles impactos de un evento lingüístico en el campo de la gravitación de Altazor primero al explorar varias aproximaciones críticas a la cuestión de la conclusión "ilegible" del poema, como función del imaginario científico que el texto comparte con ciertos descubrimientos importantes en la física teórica y experimental durante las primeras décadas del siglo XX. Al escudriñar el contexto histórico del cambio de paradigma en la física cuántica/relativista, contemporánea al momento de composición del poema, propongo examinar cómo Altazor qua texto marca y delimita el paso histórico y discursivo entre las cosmovisiones newtonianas y cuánticas. El momento de significación altazoriana, leído con respecto a las aportaciones de la física cuántica y la cosmología de la época, demuestra cómo el poema largo de Huidobro traza y toma la forma de una caída lingüística y cósmica que, no obstante, falta horizonte de expectativa y se constituye mediante procesos radicalmente heterogéneos - un rasgo del poema que llama la atención a las variaciones y los flujos cuánticos cuyas características no se pueden fijar ni predecir con certidumbre. Así, el itinerario crítico que sigue el presente ensayo no necesariamente mimetiza el "viaje en paracaídas" al que alude el título de Altazor. Al contrario, he intentado "cuantificar" y "observar" las textualidades heterogéneas y cuánticas que estructuran y al mismo tiempo desestabilizan las exploraciones poéticocosmológicas de Huidobro. La trayectoria crítica aquí emprendida parte de reflexiones sobre el análisis explícitamente lingüístico que caracteriza varios estudios críticos clave del poema, luego pasando por las estructuras cósmicas leídas a través del movimiento de la caída propuesta por el texto, por sus radicales aberraciones referenciales, y finalmente calculando el impacto de un evento traumático en las tradiciones poéticas de América Latina y de las vanguardias transatlánticas.

La cuestión del lenguaje y su colapso progresivo en Altazor no es un tema nuevo - es una idea profundamente indagada por estudiosos como Guillermo Sucre, Saúl Yurkievich, Octavio Paz, George Yúdice, Cedomil Goiç y René de Costa, entre otros. Así, las posibilidades de leer la(s) caída(s) "dentro" del lenguaje de Altazor como una fuerza mediada por una dialéctica de fracaso y triunfo es, por ende, una tendencia crítica bien evidenciada por la bibliografía amplia sobre el poema de Huidobro. Sucre, por ejemplo, no considera Altazor precisamente un poema fracasado; lo lee en términos de una voz que habla desde la misma presencia del fracaso para luego demostrar la imposibilidad de cualquier postura absoluta: 
Altazor no es un poema fracasado, sino, lo que es muy distinto, el poema del fracaso. Insisto: no sobre sino del fracaso; no un comentario alrededor del fracaso, sino su presencia misma. Uno de sus valores (y de sus riesgos, por supuesto) reside en este hecho: haber ilustrado con su escritura misma la desmesura y la imposibilidad de una aspiración de absoluto (107).

Cabe añadir que Yúdice sugiere que el nuevo lenguaje que nace de las cenizas del final del canto séptimo representa el triunfo de la polivalente palabra "caída" en su simultánea muerte y resurrección del lenguaje:

El final de Altazor, no carece de sentido; tampoco tiene solamente un sentido unívoco. En este poema se pretende resumir y superar la historia de la poesía tal como la mistifica Huidobro. De ahí las citas de código de la trascendencia vacua, del código de la ruptura y de los muchos códigos intertextuales. Al final del poema se llega al punto crítico de la creación poética; toda poesía anterior a Altazor es una aproximación asintótica a la palabra absoluta, pero este nunca llegar al absoluto es, en efecto, un fracaso. Huidobro transforma este fracaso en un triunfo; la palabra altazoriana es a la vez muerte y resurrección del lenguaje (211).

Paz también considera Altazor fracaso y triunfo simultáneo, rasgo constitutivo del momento en que "el poeta despoja paulatinamente al lenguaje de su carga de significaciones y en los últimos cantos las palabras aspiran no a significar sino a ser: sílabas que son sonajas que son semillas... El viaje por el unipacio y el espaverso de Huidobro es la historia de la ascensión del sentido al ser... [que] termina en triunfo" (12).

Por supuesto, esta lectura de Altazor está tan fundamentada en la crítica altazoriana que no habría que matizarla más, así (se espera) evitando la gota que colma el vaso. Pero en el contexto del presente ensayo, preferiría pensar las múltiples perspectivas críticas sobre las sílabas desarticuladas finales de Altazor como función de la "finalización" del poema, en vez de una posible "conclusión"”, para luego tratar las últimas enunciaciones en términos de un evento cósmico y traumático. Se puede argüir, desde una

2 Del latín "con-cludere": "abarcar, ceñir, contener" ("Concluir.” Breve diccionario etimológico de la lengua española. Ed. Guido Gómez de Silva. Ciudad de México, D. F.: Fondo de Cultura Económica, 2001.) 
postura crítica, que hay un deseo latente de orientar la experimentación poética de Altazor de acuerdo con las configuraciones temporales del alto vanguardismo, dada la fecha de composición (quizás antedatada) del texto por parte de Huidobro (1919-1931). La historiografía literaria, hasta cierto punto, demuestra la manera en que la radicalidad del proyecto poético altazoriano cierra la época de la llamada experimentación radical en las vanguardias poéticas latinoamericanas (Quiroga, "Spanish-American Poetry from 1922-1975" 314), marcando, como consecuencia, un evento poético cuyo impacto claramente se sintió en el canon latinoamericano. Sin embargo, esta observación de tipo historiográfico, por ende, revela cómo este gesto (meta) textual se estructura, en parte, como alegoría del viaje de Altazor - dada, en particular, la manera en que numerosos análisis textuales conectan la caída en el lenguaje de Altazor con la progresiva destrucción del idioma, consecuentemente orientando de manera teleológica "su" descenso como función de la progresión del Prefacio al Canto VII. Teniendo eso en cuenta, se puede sospechar de ciertas resonancias críticas de la terminología metafísica empleada por algunos estudiosos en anticipación de lo que leo como la llegada de algo completamente "otro" en el lenguaje. Ahora bien, para tomar unos ejemplos de lo que se podría considerar unas aproximaciones "metafísicas"-que no obstante constituyen aportaciones sumamente valiosas a la bibliografía sobre Huidobro- Paz sugiere que "[e]l lenguaje del canto final de Altazor ha alcanzado la dignidad suprema: la del pleno ser" (13); Yúdice describe el mito básico de Altazor en términos del milagro de "ese lenguaje antipoético y autorreferencial del último canto, lenguaje nuevo nacido de las cenizas del lenguaje destruido. Así el contexto de la literatura de ruptura no deja de tener significancia para la lectura de la obra; en efecto, Altazor pretende resumir toda esa literatura y superarla llevando el proceso de ruptura a su máxima conclusión" (184).

Un acercamiento que pensara (y hablara desde la posición de) la radicalidad de los espacios textuales de Altazor, por otro lado, se podría elaborar a través de un escrutinio de la naturaleza de lo que viene, de un evento sin horizonte de expectativa. En una discusión acertada de la futuridad "no-temporal" de lo "por venir", Jacques Derrida sugiere que:

Whenever a telos or teleology comes to orient, order, and make possible a historicity, it annuls that historicity by the same token and neutralizes the unforeseeable and incalculable irruption, the singular and exceptional alterity of what [ce qui] comes, or indeed of who 
[qui] comes, that without which, or the one without whom, nothing happens or arrives (Rogues 128).

[Siempre que un telos o una teleología, llegue a orientar, ordenar, y hacer posible una historicidad, anula al mismo tiempo esa historicidad y neutraliza la no previsible e incalculable irrupción, la alteridad singular y excepcional de lo que viene, o en efecto de quien viene, aquello sin que o sin quien no ocurra ni llegue nada ${ }^{3}$.

A pesar de la orientación de las lecturas teleológicas de Altazor, la cual subleva la caída "del" poema a una narrativa de ruina o de busca de utopía, me parece que las poéticas cosmológicas del poema quizás plantean otro escenario que sea capaz de dar cuenta de las incertidumbres lingüísticas y cuánticas presentes en el texto de Huidobro. Me refiero, más específicamente, a las múltiples "caídas" referencialmente aberrantes -lingüísticas, alegóricas y gravitacionales- que ubican a Altazor en la frontera o en el límite del paso entre la física clásica y nuestra cosmovisión cuántica actual.

Me gustaría enfocarme por el momento en el tropo de la caída y a su relación con el evento de la radical experimentación poética de Altazor. El movimiento de la caída en Altazor se inicia discursiva y temáticamente en el Prefacio, donde el protagonista toma su paracaídas y se lanza al vuelo, cayendo "de sueño en sueño por los espacios de la muerte" (55). El vínculo irreducible entre la muerte y la caída, claro, tiene resonancias bíblicas, pero también invita analogías nietzscheanas-zaratustrianas, además de evocaciones de las observaciones de Heidegger acerca de la ontología fundamental del Serpara-la-muerte del Dasein -todas lecturas bien fundamentas y establecidas en la bibliografía sobre Huidobro ${ }^{4}$. Sin embargo, en un encuentro con el Creador (justo después de comenzar el viaje) Altazor oye cómo el Creador "“[creyó] la lengua de la boca que los hombres desviaron de su rol, haciéndola aprender a hablar... a ella, ella, la bella nadadora, desviada para siempre de su rol acuático y puramente acariciador'” (56). Esta descripción de la desviación del lenguaje por parte de los hombres - una desviación del papel de lo que la fluidez oceánica supuestamente "debía” realizar- es interesante en sí, desde varias posiciones críticas, y de esta manera apoya y confirma los acercamientos críticos que tratan la desmitificación del lenguaje como

3 Las traducciones son mías.

4 Véanse de Costa, Dussuel, Goiç, Quiroga, Sucre y Yúdice, entre otros. 
función de varias rupturas lingüísticas que buscan la correspondencia perfecta y absoluta entre la palabra y la cosa-en-sí, entre significado y significante, etc. Aun así, lo que me parece más llamativo es que la cuestión de la relación tramposa del hombre con el lenguaje inmediatamente inaugure la caída de Altazor, en tanto que declare el Creador:

'creé la lengua de la boca que los hombres desviaron de su rol, haciéndola aprender a hablar... a ella, ella, la bella nadadora, desviada para siempre de su rol acuático y puramente acariciador.'

Mi paracaídas empezó a caer vertiginosamente. Tal es la fuerza de atracción de la muerte y del sepulcro abierto (56).

Esta caída vertiginosa subraya las poderosas fuerzas gravitacionales que estructuran los espacios cósmicos de Altazor, así anticipando la clasificación jerárquico-evolucionaria de suma importancia que viene al final del Prefacio:

Hombre, he ahí tu paracaídas maravilloso como el vértigo.

Poeta, he ahí tu paracaídas, maravilloso como el imán del abismo.

Mago, he ahí tu paracaídas que una palabra tuya puede convertir en un parasubidas maravilloso como el relámpago que quisiera cegar al creador $(60)$.

El paracaídas del Hombre, "maravilloso como el vértigo", enfatiza la atracción de masas que resulta de la fuerza de la gravitación, mientras la potencia magnetizada del abismo que atrae el paracaídas del Poeta desarrolla aún más las propiedades físicas de la exploración extraplanetaria. En cambio, es a través de las habilidades aparentemente creacionistas del Mago que se puede aproximar a la problemática relación entre la realidad empírica de las masas en caída y el sistema lingüístico que pretende describirlas -que claramente es una preocupación central de Altazor $^{5}$. Las operaciones verbales/ anti-gravitacionales del Mago son capaces de transformar "paracaídas" en

5 El creacionismo de Huidobro, movimiento estético vanguardista de poca trascendencia en los años 10 y 20, glorificó la actividad poética como acto de creación pura frente a las tendencias imitativas de toda tradición artística, especialmente en el contexto de la mimetización de la naturaleza. Véanse los varios manifiestos de Huidobro para las autovaloraciones del movimiento creacionista efectuadas por el mismo poeta, además de los excelentes tratamientos críticos en Vicente Huidobro y el Creacionismo (Ed. René de Costa. Madrid: Taurus, 1975). 
"parasubidas" (60) - de tal manera que sea posible invertir los efectos de la gravedad terrestre o de otros cuerpos celestes, y también la trayectoria tropológica de su caída "en" o "por" el lenguaje. Por consiguiente, Altazor, doblado significativamente en el Canto IV como "Vicente antipoeta y mago" (108), lanza su cuerpo vía de caerse hacia la profundidad del espacio celeste en una trayectoria que niega lo más fundamental de las fuerzas gravitacionales y lingüísticas.

Una posible aproximación a la confluencia de incertidumbres cosmológicas y lingüísticas en Altazor se encuentra en una serie de reflexiones teóricas acerca de la gravedad -todas observaciones e innovaciones más o menos contemporáneas al momento de la composición del poema. En términos de la diseminación de descubrimientos científicos clave con respecto a la gravedad y la relatividad en América Latina en la primera parte del siglo XX, Albert Einstein, en sus visitas a Argentina, Uruguay y Brasil en 1925, apuntó en sus diarios de viaje que no le impresionaron mucho las comunidades científicas en estos países; comentó, por lo menos, que en el ámbito argentino encontró unos cuantos físicos receptivos a sus descubrimientos (Glick, "Science in Twentieth-Century Latin America” 878-886) ${ }^{6}$. En el caso chileno, una visita del físico francés Paul Langevin precipitó una curiosidad profunda acerca de las investigaciones contemporáneas en la física cuántica y la relatividad, lo cual acompañó una serie de conferencias por los físicos chilenos Ramón Salas Edwards y Pablo Krassa sobre la teoría cuántica, la relatividad y la física experimental ${ }^{7}$. Por una parte, mientras se puede señalar que Huidobro viajaba extensivamente en Chile, Argentina, España, Francia, y Estados Unidos entre 1916 y 1931 (instalándose en varios momentos en Madrid, París, Barcelona, y Nueva York, entre otras ciudades ${ }^{8}$ ), una lectura científico-filológica

6 Eduardo L. Ortíz ha explorado la recepción de la teoría de la relatividad en Argentina -véanse sus artículos "The Transmission of Science From Europe to Argentina and its Impact on Literature: from Lugones to Borges" (Borges and Europe Revisited. Ed. Evelyn Fishburn. London: Institute of Latin American Studies, 1998) y “A Convergence of Interests: Einstein's Visit to Argentina in 1925" (Ibero-Amerkanisches Archiv 21:1-2 (1995): 67-126.)

7 Aunque no he podido calcular la profundidad del conocimiento de Huidobro acerca de estas revoluciones teóricas en las ciencias, hay bastante evidencia de una comunidad científica activa en Chile que se interesaba mucho por estos nuevos descubrimientos. Ver Gutiérrez y Gutiérrez para una discusión de la historia del desarrollo de la física en Chile desde el comienzo de la República hasta 1960.

8 Sobre la recepción de la relatividad en Europa, ver Michel Biezunski ("Einstein's Reception in Paris in 1922." The Comparative Reception of Relativity. Ed. Thomas F. Glick. 
de Altazor a la luz de la relatividad einsteiniana produce unos resultados interesantes. En el primer canto, el sujeto poético en vía de caerse describe cómo su soledad se afecta por "el paso de las estrellas que se alejan" (63), un movimiento que temáticamente evoca el aislamiento creciente de Altazor en el firmamento que retrocede, pero también sugiere algún conocimiento de la expansión del universo. La noción de la expansión y la inflación cósmica -basándose en unas soluciones particulares a las ecuaciones de campo de la relatividad desarrolladas por Einstein (1915), matemáticamente aprobadas por Alexander Friedman (1922), y confirmadas en los experimentos de Edwin Hubble (1929) - cuenta con la flexibilidad del espacio y el tiempo para explicar que es el espacio mismo (curvado por la presencia de masas gravitacionales) lo que está en expansión (Greene 229-233). Este cambio radical a nuestra cosmovisión es significativo, desde que utiliza la teoría general de la relatividad para explicar la simultánea expansión del tiempo y del espacio (como espacio-tiempo) para enfatizar la imposibilidad de cualquier centro fijo o eje que originara la expansión (Greene 232). Siguiendo este hilo de pensamiento, se puede pensar la urgente y repetida insistencia del "No hay tiempo que perder" en el Canto IV como un fenómeno cósmico que situara la caída de Altazor justo en el medio del cambio de paradigma del auge de la relatividad einsteiniana en los años veinte. De esta manera, el poema atestigua la noción de la dilación del tiempo para los objetos en moción relativa, lo cual demuestra, ya que "no hay tiempo que perder", la necesidad de "[jugar] fuera del tiempo" (118); es decir, "fuera" del tiempo, ya que el tiempo mismo forma parte del destiempo (a)temporal del flujo de nuestra cosmovisión/consciencia pos-relativista. En suma, Altazor trata la cuestión de la relatividad al subrayar la imposibilidad de una noción absoluta del tiempo y del espacio, compartida por todo observador, escenario en que no puede existir ninguna medida incondicional de la temporalidad a escala universal.

Otro componente clave de esta "revolución científica", como la clasifica el filósofo de la ciencia Thomas Kuhn, se puede encontrar en otros aportes y debates en la física teórica y experimental en la primera parte del siglo $\mathrm{XX}^{9}$.

Dordrecht, Holland: D. Reidel Publishing Company, 1987) y Thomas F. Glick (Einstein in Spain: Relativity and the Recovery of Science. Princeton: Princeton UP, 1998).

9 Para una discusión de la naturaleza de cambios de paradigma en el pensamiento científico, ver las obras seminales de Kuhn: The Copernican Revolution: Planetary Astronomy 
Hay que notar que estos descubrimientos no precipitaron solo un cambio en el pensamiento contemporáneo en un contexto científico-académico; por el contrario, como sugiere Alicia Rivero, "Einstein's relativity and the Copenhagen interpretation of quantum mechanics presented a new worldview, which reconceptualised time, space and other aspects of classical physics, while metamorphosing the art, literature and philosophy of the twentieth century" (137) [La relatividad einsteiniana y la interpretación de Copenhague de la mecánica cuántica presentaron una nueva cosmovisión que reconceptualizó el tiempo, el espacio, y otros aspectos de la física clásica, al mismo tiempo que metamorfoseó el arte, la literatura, y la filosofía del siglo $\mathrm{XX}]^{10}$. En términos más dramáticos, Wassily Kandinsky, fundador del arte abstracto, sostuvo que en el contexto del derrocamiento del modelo clásico del universo, "[t]he collapse of the atom model was equivalent, in my soul, to the collapse of the whole world. Suddenly the thickest walls fell. I would not have been amazed if a stone appeared before my eye in the air, melted, and became invisible" (Cit. en Randall 117) [El colapso del modelo del átomo era equivalente, en mi alma, al colapso del mundo entero. De repente todo muro impasible fue derrumbado. No habría estado asombrado si una piedra hubiera aparecido en el aire delante de mis ojos, se hubiera derretido, y luego se hubiera convertido en algo invisible]. Los comentarios de Kandinsky acerca de la física cuántica son un poco hiperbólicos, pero es verdad que hubo unos aportes extraños (que Einstein llamaba “spooky") durante la época. En particular, la interpretación generalmente aceptada de la mecánica cuántica - llamada la Copenhague y basada en el principio de incertidumbre de Werner Heisenberg, el cual sugiere que hay límites a la exactitud con que la posición y el momento de una partícula pueden medirse-junto con las investigaciones de Niels Bohr sobre la dualidad ola-partícula de la luz, pusieron en tela de juicio la certeza que ostensiblemente garantizaba la cosmovisión clásica.

En el universo newtoniano -cuyas leyes de la moción todavía describen de manera muy adecuada el movimiento de objetos no masivos y de baja velocidad- se habría podido describir la trayectoria de la caída de Altazor, por ejemplo, dada suficiente información sobre todo cuerpo extraplanetario y

in the Development of Western Thought (Cambridge: Harvard UP, 1957) y The Structure of Scientific Revolutions (Chicago: U. of Chicago P., 1962).

10 N. Katherine Hayles data el momento clave del desarrollo de la mecánica cuántica al año 1927, cuando Werner Heisenberg formalizó el principio de incertidumbre (The Cosmic Web 43). 
partícula involucrados. En el contexto de este cambio de paradigma, una de las cuestiones fundamentales en juego en el sistema de partículas en interacción en el flujo celeste altazoriano está vinculada a la incertidumbre al nivel molecular, descrita por el llamado "efecto-mariposa" en la teoría del caos ${ }^{11}$. Esta es una perspectiva que examina la suposición de que las condiciones iníciales de un sistema (e.g., un cambio minúsculo en el viento como efecto del batir de alas de una mariposa) puedan radicalmente influir patrones del tiempo (así causando, por ejemplo, un torbellino en el otro lado del mundo). El físico Brian Greene subraya una analogía meteorológica bastante parecida en la cosmovisión newtoniana, dado que según el universo clásico, "if we knew in complete detail the state of the environment (the positions and velocities of every one of its particulate ingredients), we would be able to predict (given sufficient calculational prowess) with certainty whether it will rain at 4:07 p.m. tomorrow..." (91) [si supiéramos completamente toda información posible sobre el ambiente (las posiciones y las velocidades de cada una de sus partículas), podríamos predecir (dada suficiente sofisticación en los cálculos) con certeza si llueve mañana a las 16:07...]. Me parece que esta reflexión teórica comparte cierto imaginario científico con las exploraciones sobre las correspondencias cósmicas en el Canto IV de Altazor, con respecto a la manera en que el texto examina los límites epistemológicos, poéticos y probabilísticos de la certeza en un mundo cuántico. Escribe Huidobro:

Qué hace la golondrina que vi esta mañana

¿Firmando cartas en el vacío?

Cuando muevo el pie izquierdo

¿Qué hace con su pie el gran mandarín chino?

Cuando enciendo un cigarro

¿Qué hacen los otros cigarros que vienen en el barco?

¿En dónde está la planta del fuego futuro?

Y si yo levanto los ojos ahora mismo

¿Qué hace con sus ojos el explorador de pie en el polo?

Yo estoy aquí

¿En dónde están los otros?

Eco de gesto en gesto

11 Ver N. Katherine Hayles para una discusión acertada y clara de la dinámica no linear de la meteorología, en tanto que los cambios pequeños y los flujos en sistemas no lineares puedan tener grandes efectos (Chaos Bound 12). 


\section{Cadena electrizada o sin correspondencias \\ Interrumpido el ritmo solitario \\ ¿Quiénes se están muriendo y quiénes nacen \\ Mientras mi pluma corre en el papel? (101)}

Esta indagación trágico-cómica (o por lo menos idiosincrática) de causa y efecto interroga la certeza del mundo clásico, pero al mismo tiempo intercambia la vinculada "cadena electrizada" por un mundo "sin correspondencias". Este gesto desplaza la atención crítica del lector a la manera en que dicho ritmo solitario se interrumpe, en vez de constituirse como ritmo en sí, de modo que parodie poemas como el soneto famoso "Correspondences" del poeta francés Charles Baudelaire, además del énfasis temático y discursivo en la armonía en la obra de poetas latinoamericanos modernistas como José Martí y, en particular, Rubén Darío. Es decir, esta noción no solo denota una cosmovisión en que la posición y el momento de cierta partícula pueden expresarse exclusivamente en términos de una ola de probabilidad -y no unas coordenadas específicas- sino también la especie de entrelazamiento cuántico tan odiado por Einstein ${ }^{12}$. El físico alemán protestaba repetidamente contra lo que él llamaba "acción fantasmal a distancia" ("spooky action at a distance"), fenómeno previsto por la física cuántica que postula la existencia de partículas entrelazadas a pesar de las grandes distancias que las separan. Decir que dos partículas son "entrelazadas" describe una manifestación en la que dos entidades sub-atómicas inicialmente idénticas (como, por ejemplo, electrones o fotones), al separarlas a grandes distancias, las dos todavía inevitablemente demuestran las mismas propiedades al actuar solamente sobre una de ellas -a pesar del límite "último" de la velocidad de la luz para transmitir información. Este principio contra-intuitivo influye el tipo de correspondencias irónicas exploradas en este Canto IV, en estos actos miméticos que sin embargo están simultáneamente desconectados el uno del otro.

12 El experimento famoso "EPR" (Einstein-Podalsky-Rosen, 1935) sobre la posible incompatibilidad de la incertidumbre cuántica con la concepción clásico-newtoniana del universo es el momento histórico clave en este debate. No obstante, su estudio revolucionario resultó contribuir al desarrollo de la misma mecánica cuántica que intentaba criticar-de formas complejas y variadas que eventualmente contradirían a Einstein frente al debate cuántico. En este contexto, es relevante la teoría de John S. Bell (1964), la que puso fin a las formulaciones posiblemente incompletas de la mecánica cuántica propuestas por EPR. 
La caída multifacética de Altazor, pues, subraya los lacunae que separan las cosmovisiones newtonianas y cuánticas. Las raíces pos-clásicas de Altazor recalcan las estructuras cosmológicas y matemáticas necesariamente "fantasmales" en el espacio celeste del poema, destacando la naturaleza no-anticipada e incierta de la caída materializada en el viaje textual de siete cantos. Mi insistencia en lo que no se puede anticipar, en lo que viene sin horizonte de expectativa, es un producto de mi convicción de que las lecturas que intentan dar cuenta del evento textual de Altazor al construir un edifico teleológico en donde basarse no necesariamente consideran los flujos cuánticos y radicalmente singulares que llegan al final del poema. Inscribir la caída de Altazor en el contexto de las incertidumbres de una poética cuántica, sin embargo, trata la naturaleza radical del evento en términos de su propia llegada no-anticipada, así razonando el movimiento fundamentalmente imprevisible de la caída sin necesidad de unirse a una posicionalidad "clásica" ni teleológica. De esta forma, me parece que una lectura "newtoniana" de Altazor se constituiría como producto de las raíces teleológicas que borraran la singularidad de lo por venir, de lo que llega en las sílabas desarticuladas de la "conclusión" del poema. Mi lectura "cuantizada" de la trayectoria de la caída de Altazor, por otro lado, señala dónde el poema "acaba" en sus múltiples incertidumbres atómicas, cosmológicas, y lingüísticas.

Una "muestra" o "medida" específica del impacto meta-lingüístico, discursivo, y cosmológico de Altazor se puede calcular en la descomposición verbal y química que registra el evento de la trayectoria celeste del meteoro en el Canto IV:

Aquí yace Carlota ojos marítimos

Se le rompió un satélite

Aquí yace Matías en su corazón dos escualos se batían

Aquí yace Marcelo mar y cielo en el mismo violoncelo

Aquí yace Susana cansada de pelear contra el olvido

Aquí yace Teresa esa es la tierra que araron sus ojos hoy ocupada por su cuerpo

Aquí yace Angélica anclada en el puerto de sus brazos

Aquí yace Rosario río de rosas hasta el infinito

Aquí yace Raimundo raíces del mundo son sus venas

Aquí yace Clarisa clara risa enclaustrada en la luz

Aquí yace Alejandro antro alejado ala adentro

Aquí yace Gabriela rotos los diques sube en las savias 
hasta el sueño esperando la resurrección

Aquí yace Altazor azor fulminado por la altura

Aquí yace Vicente antipoeta y mago

Ciego sería el que llorara

Ciego como el cometa que va con su bastón

Y su neblina de ánimas que lo siguen

Obediente al instinto de sus sentidos

Sin hacer caso de los meteoros que apedrean desde lejos

$Y$ viven en colonias según la temporada

El meteoro insolente cruza por el cielo

El meteplata el metecobre

El metepiedras en el infinito

Meteópalos en la mirada (108-109).

Esta serie cosmológica surge como resultado de unas desconstrucciones lingüísticas, seguidas por la fragmentación progresiva de unos nombres propios - de Marcelo a "mar" y “cielo", de Clarisa a "clara” y "risa”, además de Alejandro a "antro" y "alejado", entre otros-y también revela los orígenes del nombre de Altazor como función de lo celeste y lo aviario, de "altura" y "azor". Ahora bien, es interesante notar que mientras la descomposición de los nombres inicia ecos aurales y tartamudeos visuales a través de una operación paranomasística, el único nombre no desconstruido es el de "Vicente", una clara invocación del mismo Huidobro, antipoeta cuyo verso creacionista no deja ni huella ni eco. Al vincular su propia destreza poética con la del mago (así como en el Prefacio), se puede ver cómo la mirada antipoética intenta resistir el tipo de operaciones desconstructivas a las que los otros nombres son sujetos. No obstante, la progresión desde lo propio a lo común en este pasaje -de "Marcelo" a "meteoro", por ejemplo- solo logra dramatizar la manera en que el nombre propio en sí necesariamente funciona con respecto al mismo sistema de diferenciales que estructura y también desestabiliza la lengua. Como sugiere Geoffrey Bennington en el contexto del trabajo crítico de Jacques Derrida,

[The proper name] is the keystone of logocentrism... What is called by the generic common noun 'proper name' must function, it too, in a system of differences: this or that proper name rather than another designates this or that individual rather than another and thus is marked by the trace of these others, in a classification (GL, 86b, 137a), if only a two-term classification...For there to be a truly proper name, 
there would have to be only one proper name, which would then not even be a name, but pure appellation of the pure other, absolute vocative (cf. EO, 107-8; GR, 110-1; WD, 105), which would not even call, for calling implies distance and différance, but would be proffered in the presence of the other, who would in that case not even be other...(105)

[[El nombre propio] es la piedra clave del logocentrismo...Lo que se denomina "nombre propio" (a través del empleo de un sustantivo genérico y común) también tiene que participar en un sistema de diferencias: este o aquel nombre propio en vez de otro así marcado por la huella de estos otros, en una clasificación (GL, 86b, 137a), si solo una clasificación a base de dos términos...La existencia de un nombre propio digno de su nombre requeriría la existencia de solo un nombre propio, el que entonces no sería ni siquiera nombre sino apelación pura a lo puramente otro, vocativo absoluto (cf. EO, 107-8; GR, 110-1; WD, 105), el que no apelaría, desde que el apelar implica distancia y différance, sino que sería ofrecido en la presencia del otro, quien en ese caso aun no sería otro...]

Este acto (im)propio de nombrar manifiesta el descenso paralelo del nombre y los objetos celestes tales como cometas, estrellas, y posiblemente más significativamente en este caso, meteoros. En tanto que los juegos lingüísticos lúdicos de Altazor permitan el tratamiento material de "meteoro" como si fuera una combinación de "mete" (del verbo "meter") y "oro", lo que "se produce" aquí representa una serie de reacciones químico-verbales que añaden "plata", "cobre", "piedras" más genéricas, y finalmente, "ópalos". René de Costa comenta esta trasposición en términos de su efecto cómico - “[b]ut effects are not causes, and here humor functions to point up the polyvalent nature of language, its potential for generating meanings according to context" (Vicente Huidobro: The Careers of a Poet 156) [pero efectos no son causas, y aquí el humor sirve para señalar la naturaleza polivalente del lenguaje, su potencia de generar sentidos según el contexto]- pero en realidad su efecto cósmico demuestra cómo lo lingüístico y lo cosmológico no convergen según las leyes naturales. Es decir, estos mismos "meteoros/meteplata/metecobre/ metepiedras/meteópalos" están cayendo, pero no necesariamente hacia la tierra como función de la atracción gravitacional entre cuerpos, sino en el lenguaje.

Desde el punto de vista referencial, este ejemplo del "meteoro" revela una serie de convergencias muy llamativas entre la fantasmalidad cuántico- 
cosmológica de Altazor y la cuestión de la "literariedad" constitutiva del poema ${ }^{13}$. El cambio de paradigma que denominó el cambio desde lo que se podría llamar la escritura "clásica" al tipo de poética cosmológico-cuántica en juego en Altazor refleja la manera en que el lenguaje en sí ya siempre estrena y ensaya estas incertidumbres. Desde la perspectiva de la teoría literaria, Paul de Man, en un tratamiento particularmente sugerente de la incertidumbre lingüística, describe la relación tensa entre la realidad empírica del mundo material y la materialidad del lenguaje al enfatizar la manera en que

[1]iterature is fiction not because it somehow refuses to acknowledge 'reality,' but because it is not a priori certain that language functions according to principles which are those, or which are like those, of the phenomenal world. It is therefore not a priori certain that literature is a reliable source of information about anything but its own language...(11)

[la literatura es ficción no porque de algún modo se niega a reconocer 'la realidad', sino porque no está a priori segura que el lenguaje funciona según los principios del mundo fenomenal o algunos principios que son como ellos. Así no está segura a priori que la literatura sea una fuente fiable de información sobre algo más que su propio lenguaje...]

De Man nos avisa sobre los peligros inherentes en confundir "the materiality of the signifier with the materiality of what it signifies" [la materialidad del significante con la materialidad de lo que significa] desde que "no one in his right mind will try to grow grapes by the luminosity of the word 'day"' (11) [ninguna persona cuerda intentaría cultivar uvas utilizando la luminosidad de la palabra 'día']. La distinción que establece de Man entre la realidad empírica del mundo físico y la materialidad del lenguaje está expresada, claro, de manera un poco cómica, pero sin embargo señala cómo la aproximación al mundo a través del lenguaje -su "medida", digamos- es indicativa de la imprecisión de dicho "cálculo". Hasta cierto punto, las múltiples "caídas"

13 Alicia Rivero describe algunas de las cuestiones que vinculan la incertidumbre cuántica con lo que ella llama "la ficción cuántica" en "Heisenberg’s Uncertainty Principle in Contemporary Spanish American Fiction" (Science and the Creative Imagination in Latin America. Ed. Evelyn Fishburn and Eduardo L. Ortiz. London: Institute for the Study of the Americas, 2005). 
que (metafóricamente) abarcan los intersticios y los deslizamientos propios al lenguaje así pueden leerse a la luz de los vínculos entre lo cuántico y lo lingüístico en Altazor. Al mismo tiempo, no quiero sugerir que hubo alguna vez un momento de correspondencia edénica y perfecta entre la palabra y la cosa-en-sí - lo cual sí forma parte del imaginario de la caída alegórica que ha sido leída en el contexto de Altazor, en cuanto a la búsqueda posible de un lenguaje puro. Aun así, creo que una aproximación útil a las caídas multifacéticas presentes en las exploraciones cuánticas de Altazor se puede encontrar en una lectura detenida del impacto traumático de múltiples eventos lingüísticos y gravitacionales en el poema de Huidobro.

En un ensayo sobre los tropos de la caída en los escritos de Paul de Man, Cathy Caruth nos ofrece un marco teórico para evaluar cómo la relación entre el mundo fenomenal y el lenguaje en la cosmovisión newtoniana se hizo problemática - un punto de vista que, como hemos visto, daría lugar a crises de significación posteriores a la luz de la relatividad y la física cuántica. Caruth describe cómo la lectura demaniana de las marionetas antigravitacionales de Heinrich von Kleist en el "Über das Marionettentheater" estrena una disyunción clave que distingue las propiedades referenciales del lenguaje de la materialidad fenomenal que pertenece a las representaciones matemáticas de la ley de la gravedad en las ecuaciones de Newton: “.... with the introduction of gravitation, the only thing that was adequate to the world was, paradoxically, that which didn't refer (mathematics); and what did refer, language, could no longer describe the world. In a world of falling, reference could not adequately describe the world" (Caruth 76) [...con la introducción de las leyes de la gravitación, lo único que describía el mundo era, paradójicamente, algo que no refería (las matemáticas); y lo que sí refería, el lenguaje, ya no pudo describir el mundo. En un mundo de caídas, la referencia no pudo describir el mundo adecuadamente]. Las marionetas de Kleist bailan de tal modo que son capaces de eludir el problema de la referencialidad "in a formal, quantified system that is as predictable, and ultimately nonspecific -or nonreferential- as a mathematics" (81) [en un sistema formal y cuantificado que es tan predecible, y últimamente no-específico -o no referencial-como una matemática]. Donde Caruth lee la lectura demaniana en términos de la fuerza performativa de la manifestación del lenguaje como materialidad, ella observa que

philosophy must, and yet cannot, fully integrate a dimension of language that not only shows, or represents, but acts...It is paradoxically in this deathlike break, or resistance to phenomenal knowledge, 
that the system will encounter the resistance, de Man suggests, of reference (87).

[la filosofía necesita, pero no puede, completamente integrar una dimensión del lenguaje que no sólo demuestra o representa, sino que actúa...Es paradójicamente esta ruptura mortal, o la resistencia al conocimiento fenomenal, donde el sistema encontrará, sugiere de Man, la resistencia de la referencia.]

Las rupturas y "discontinuidades" en la referencia, en realidad, son lo que permite el desarrollo del sentido cuando "force disarticulates the system as it attempts to distinguish and unify empirical and conceptual discourse, that is, to know itself as independent of empirical referents" (88) [la fuerza desarticula el sistema al mismo tiempo que intenta distinguir y unificar discurso empírico y conceptual, es decir, conocerse como independiente de referentes empíricos]. Estas caídas, representadas lingüísticamente de manera inadecuada, muestran cómo la teoría y la lectura así son el movimiento de la caída en sí, propagada por esta fuerza que materializa la resistencia a, o de, la referencia.

La disyunción entre las ecuaciones que buscan describir el movimiento de objetos que caen y los elementos lingüísticos que los describen subraya la incongruencia alegórica que "rige" la manera en que el lenguaje funciona. Pero tal como atestiguan los descubrimientos en la teoría cuántica de la primera parte del siglo XX -sin mencionar el trabajo científico actual en varios campos de investigación, como, por ejemplo, la teoría de (super) cuerdas, la teoría $\mathrm{M}$, la gravedad cuántica de lazos, la teoría de twistores, etcétera- la fuerza atractiva de la gravedad, tal como la describió Newton, no explica de manera exacta lo que pasa a los cuerpos en movimiento. El mero peso discursivo de descubrimientos como la teoría de relatividad de Einstein, el principio de incertidumbre de Heisenberg y la dualidad ola-partícula de la luz, entre otros, señalan el cambio de paradigma del universo clásico a la cosmovisión cuántica/posrelativista que, como hemos visto, se registra como evento en la radical textualidad poética de Altazor. Parte esencial del evento traumático de Altazor -cuyo impacto resonó estruendosamente en el canon poético latinoamericano- es la llegada famosa (infame) de las sílabas desarticuladas y últimas en el Canto VII, en donde Altazor registra sus caídas y flujos "finales", sus gritos sofocados y sus agonías, sus anhelos de la resurrección y su imaginario-fénix (siguiendo a Paz, a Yúdice, et al.). A la luz de nuestra lectura cuántico-cosmológica, podríamos (volver a) considerar 
cómo "acaba" el poema en sus articulaciones quizás no-referenciales -y no necesariamente lo que "significa" ni lo que "concluye" aquí (en el sentido etimológico de la palabra):

Semperiva ivarisa tarirá

Campanudio lalalí Auriciento auronida

Lalalí io ia

i i i o

Ai a i ai a i i i i o ia (138).

En vez de poner fin a la significación a través de un proceso hermenéutico que descifrara fragmentos de palabras en este pasaje - el cual nos llevaría a topografías y texualidades áureas (dada la repetición del prefijo "aur-"), cómicas ("-risa"), y la eternidad ("semper"), para señalar solo tres ejemplos -podríamos decir que la organización visual de estos versos se parece a la prosodia en español, y también "termina" con una cadena de vocales que se aproxima a cierto tipo de articulación gutural y vocalizada. Pero en el contexto de las incertidumbres cuánticas de Altazor, estos versos finales pueden leerse como una "muestra" observada de los flujos cuánticos del poema-es decir, una selección entre posibles combinaciones de elementos lingüísticos (letras, vocales acentuadas y espacios, desplegados en un arreglo visual particular en la página) que registran cambios de "energía” en el sistema lingüístico cuyas probabilidades pueden calcularse con respecto al principio de incertidumbre de Heisenberg ${ }^{14}$. Así, en el contexto de la cuantización de la palabra poética en Altazor (ahora "medida" y "observada"), estos mismos puntos "finales" son representativos del tipo de límites y bordes que desestabilizan las fronteras discursivas del mismo poema, desde que es un texto que anhela, paradójicamente, "[medir] paso a paso el infinito" (99) y ver "[m]ás allá del último horizonte" (100), así cuestionando la experiencia-límite de la frontera en sí. Estos bordes solo son bordes, hasta cierto punto, si ya siempre

14 Estos flujos cuánticos, hasta cierto punto, anticipan el contenido de la biblioteca infinita del cuento archi-conocido de Borges, "La biblioteca de Babel" (entre otros textos fantásticos y metafísicos del maestro argentino). 
se transgreden sin horizonte de expectativa ${ }^{15}$-y de ahí surge lo fantasmalcuántico de la poética textual-cósmica de Altazor y lo que viene sin horizonte en el canto "terminal". Despojado de una metafísica de la presencia que negara la radicalidad de este evento lingüístico no-comunicativo, la medida cuántica del espacio textual de Altazor así alude al impacto traumático de este "final" problemático, este momento no referencial -textual y (meta)crítico, y también en el escenario de las vanguardias mundiales y el cambio radical en la cosmovisión que resultó de los nuevos descubrimientos en la física y la cosmología. Y, últimamente, lo que facilita nuestra lectura cuántica de la poesía de Huidobro es un acercamiento a lo que llega en los flujos finales e inciertos de Altazor: cierto mapeo de un "camino" particular, esencialmente una compleja función de onda verbal que "opta" por cierta "finalidad" específica entre una miríada de posibilidades.

\section{BIBLIOGRAFÍA}

Bary, David. Huidobro o la vocación poética. Granada: Universidad de Granada, 1963.

Bennington, Geoffrey and Jacques Derrida. Jacques Derrida. Chicago: U of Chicago P, 1993.

Biezunski, Michel. "Einstein's Reception in Paris in 1922.” The Comparative Reception of Relativity. Ed. Thomas F. Glick. Dordrecht, Holland: D. Reidel Publishing Company, 1987.

Caruth, Cathy. Unclaimed Experience: Trauma, Narrative, and History. Baltimore: Johns Hopkins UP, 1996.

"Concluir". Breve diccionario etimológico de la lengua española. Ed. Guido Gómez de Silva. Ciudad de México, D. F.: Fondo de Cultura Económica, 2001.

Costa, René de. En pos de Huidobro. Siete ensayos de aproximación. Santiago de Chile: Editorial Universitaria, 1978.

Vicente Huidobro: The Careers of a Poet. London: Oxford UP, 1984.

ed. Vicente Huidobro y el Creacionismo. Madrid: Taurus, 1975.

De Man, Paul. The Resistance to Theory. Minneapolis: U of Minnesota P, 1986.

Derrida, Jacques. Positions. Trans. Alan Bass. Chicago: The U of Chicago P, 1981.

Rogues: Two Essays on Reason. Trans. Pascale-Anne Brault and Michael Naas. Stanford: Stanford University Press, 2005.

Dussuel, S. J. Francisco. "El creacionismo y la inquietud de lo infinito". Atenea 130/379 (enero-febrero-marzo, 1958): 92-131.

15 Este argumento derrideano se encuentra en varios escritos del filósofo francés -véanse en particular Positions y Rogues, entre otros textos. 
Glick, Thomas F. Einstein in Spain: Relativity and the Recovery of Science. Princeton: Princeton UP, 1998.

"Science in Twentieth-Century Latin America." The Cambridge History of Latin America. Vol XI. Ed. Leslie Bethell. Cambridge: Cambridge UP, 1995. 878-886.

Goiç, Cedomil. La poesía de Vicente Huidobro. Santiago: Ediciones Nueva Universidad, Instituto de Letras de la Universidad Católica de Chile, 1974.

Greene, Brian. The Fabric of the Cosmos: Space, Time, and the Texture of Reality. New York: Vintage Books, 2005.

Gutiérrez, Claudio and Flavio Gutiérrez. "Physics: Trajectory in Chile.” Trans. Cristina Labarca. Historia (Santiago) 39/2 (jul/dic 2006): 477-496. http://socialsciences.scielo.org/scielo.php?script=sci_arttext\&pid=S0717-71942006000200001\&lng=en\&nrm=iso

Hayles, N. Katherine. Chaos Bound: Orderly Disorder in Contemporary Literature and Science. Ithaca: Cornell UP, 1990.

The Cosmic Web: Scientific Field Models and Literary Strategies in the Twentieth Century. Ithaca: Cornell UP, 1984.

Huidobro, Vicente. Altazor. Temblor de cielo. Ed. René de Costa. Madrid: Cátedra, 2000.

Kuhn, Thomas S. The Copernican Revolution: Planetary Astronomy in the Development of Western Thought. 1957. Cambridge: Harvard UP, 1992.

The Structure of Scientific Revolutions. 1962. Chicago: U of Chicago P, 1996.

Ortiz, Eduardo. “A Convergence of Interests: Einstein's Visit to Argentina in 1925." IberoAmerkanisches Archiv 21/1-2 (1995): 67-126.

"The Transmission of Science from Europe to Argentina and its Impact in Latin America: From Lugones to Borges." Borges and Europe Revisited. Ed. Evelyn Fishburn. London: Institute of Latin American Studies, 1998. 108-23.

Paz, Octavio. "Decir sin decir." Vuelta 107 (octubre 1985): 12-13.

Quiroga, José. “El entierro de la poesía: Huidobro, Nietzsche, y Altazor.” MLN 107.2 (March 1992): 342-362.

"Spanish-American Poetry from 1922-1975." The Cambridge History of Latin American Literature. Ed. Roberto González-Echevarría and Enrique Pupo-Walker. Vol. 2. Cambridge: Cambridge UP, 1996. 303-364.

Randall, Lisa. Warped Passages: Unraveling the Mysteries of the Universe's Hidden Dimensions. New York: Harper Collins, 2005.

Rivero, Alicia. "Heisenberg's Uncertainty Principle in Contemporary Spanish American Fiction." Science and the Creative Imagination in Latin America. Ed. Evelyn Fishburn and Eduardo L. Ortiz. London: Institute for the Study of the Americas, 2005. 129-150.

Sucre, Guillermo. La máscara, la transparencia. Ensayos sobre poesía hispanoamericana. México: Tierra Firme, 1985.

Yúdice, George. Vicente Huidobro y la motivación del lenguaje. Buenos Aires: Editorial Galerna, 1978.

Yurkievich, Saúl. Fundadores de la nueva poesía latinoamericana. Barcelona: Barral, 1973. 
